\title{
A potential new tool for cardiovascular disease prevention
}

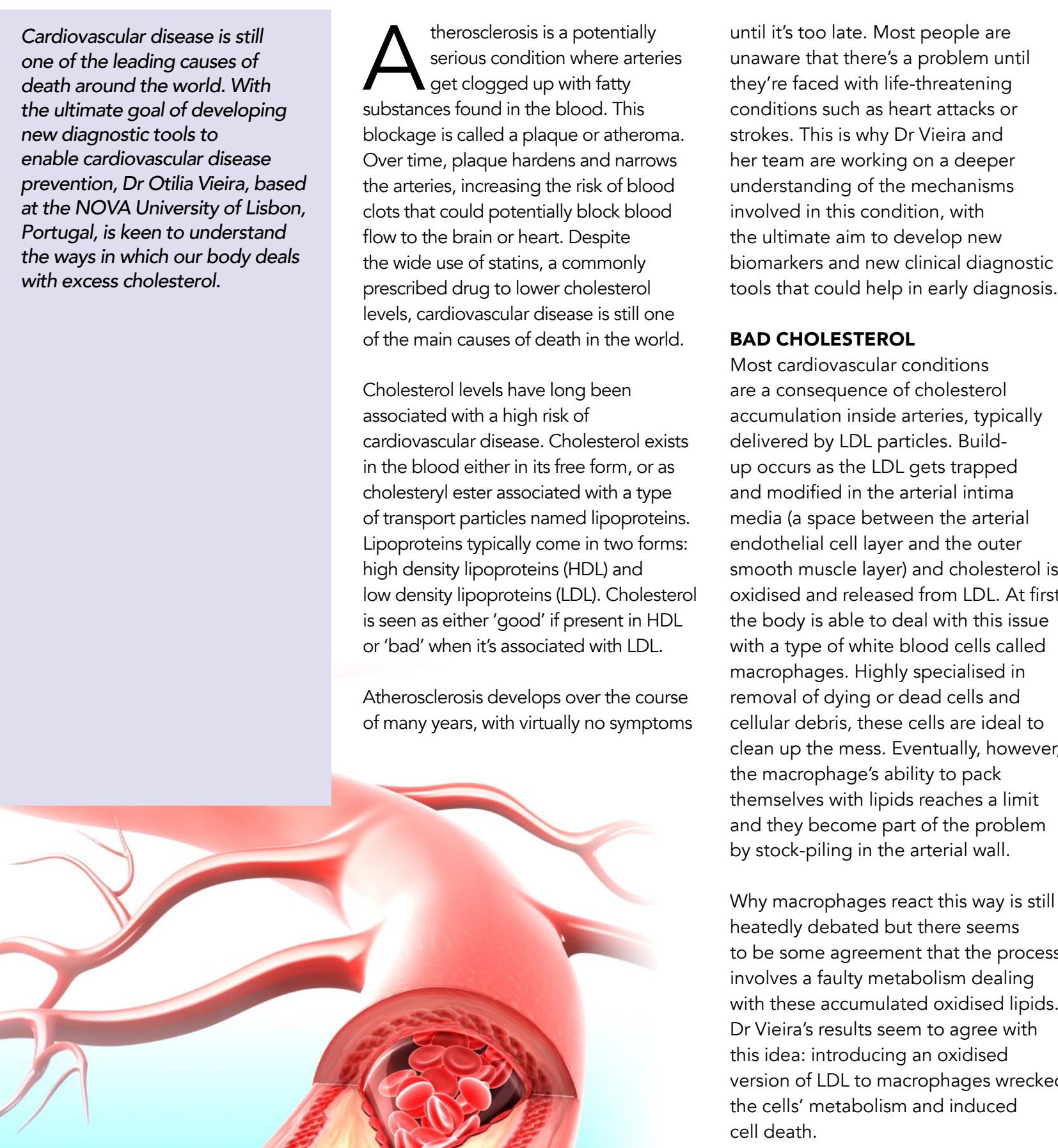

Cardiovascular disease is still one of the leading causes of death around the world. With the ultimate goal of developing new diagnostic tools to

preve

at the NOVA University of Lisbon, Portugal, is keen to understand the ways in which our body deals with excess cholesterol.

therosclerosis is a potentially
serious condition where arterie get clogged up with fatty substances found in the blood. This Over time, plaque hardens and narrows the arteries, inceasing the risk of blows clots that could potentially block blood flow to the brain or heart. Despite the wide use of statins, a commonly prescribed drug to lower cholesterol levels, cardiovascular disease is still on of the main causes of death in the world

Cholesterol levels have long bee associated with a high risk of

cardiovascular disease. Cholesterol exists in the blood either in its free form, or as cholesteryl ester associated with a type of transport particles named lipoproteins. Lipoproteins typically come in two form high density lipoproteins (HDL) and low density lipoproteins (LDL). Cholesterol is seen as either 'good if present inDL

Atherosclerosis develops over the course of many years, with virtually no syn

until it's too late. Most people are unaware that there's a problem unt they re faced with life-threatening conditions such as heart attacks or strokes. This is why Dr Vieira and understanding of the mechanisms involved in this condition, with the ultimate aim to develop new biomarkers and new clinical diagnostic tools that could help in early diagnosis.

BAD CHOLESTEROL Most cardiovascular conditions ccumulation inside arteres, typic delivered by LDL particles. Buildup occurs as the LDL gets trapped and modified in the arterial intima media (a space between the arterial endothelial cell layer and the outer smooth muscle layer) and cholesterol is oxidised and released from LDL. At first, with a type of white beal with this issue macrophages Highly specialised in removal of dying or as cells and cellular debris, these cells are ideal to clean up the mess, Eventually, however, the macrophage's ability to pack themselves with lipids reaches a limit and they become part of the problem by stock-piling in the arterial wall.

Why macrophages react this way is still heatedly debated but there seems to be some agreement that the process involves a faulty metabolism dealing with these accumulated oxidised lipids. Dr Vieira's results seem to agree with this idea. introducing an oxidised version of LDL to macrophages wrecked cell death.
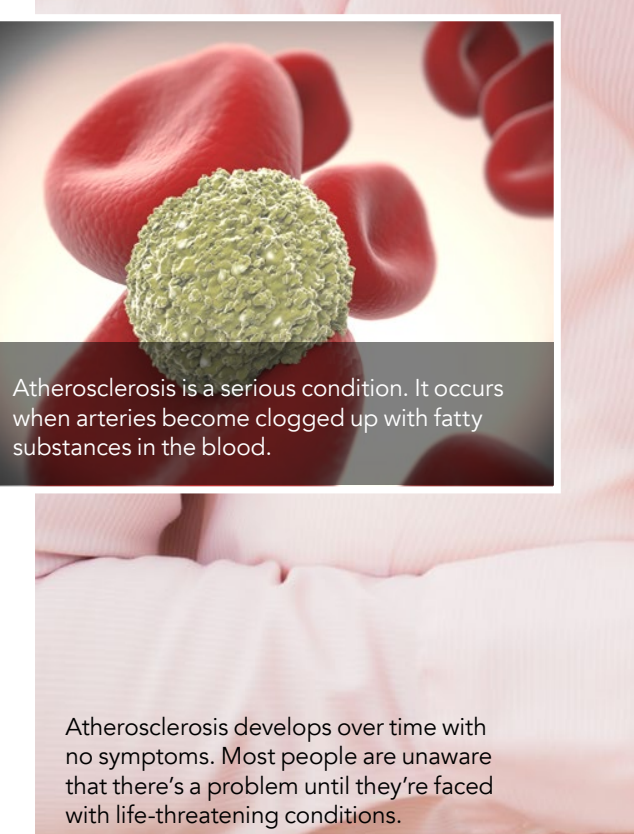

Under normal circumstances, these cells would have been expected to convert safer for storage. However, cells exposed to this treatment were clearly unable to continue their normal metabolism and the modified LDL was simply allowed to accumulate. Inevitably, this led to irreversible and uncontrolled lipid accumulation and eventually cell death.

This cascade of events seems to be caused by a break-down in the way the cell handles the excess of lipids accumulating inside. DrVieira and her team found that the main area affected was the lysosome, where incoming substances are stored. The problem was that lipids kept coming in, but the lysosome was in, but the lysosom
up the pace.

"We have shown, for the first time, that a single product of the oxidation of the low density lipoproteins is sufficient to induce both the initiation of atheroma formation and exacerbate the pathology", says Dr Vieira. The team hope this work will be crucial in future studies dedicated to cardiovascular disease prevention, by leading to the identification of new therapeutic agents

GOOD CHOLESTEROL As described earlier, while LDL

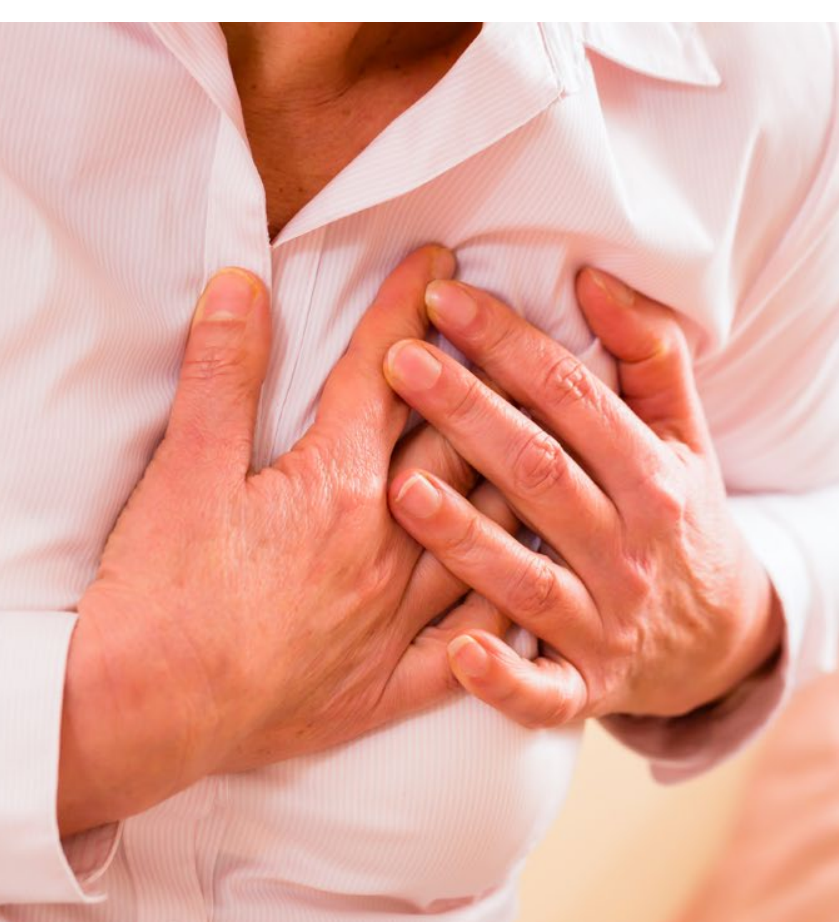

Despite the wide use of statins, a commonly prescribed drug to lower cholesterol levels, cardiovascular disease is still one of the main causes of death in the world.

and its oxidised derivatives can drive atherosclerosis, HDL are commonly perceived as having the opposite effect. It is generally accepted that the process started by LDL gets worse if there isn' and carry it back to the liver.

While both ideas have a strong scientific backing, the exact mechanisms explaining how HDL operates are still

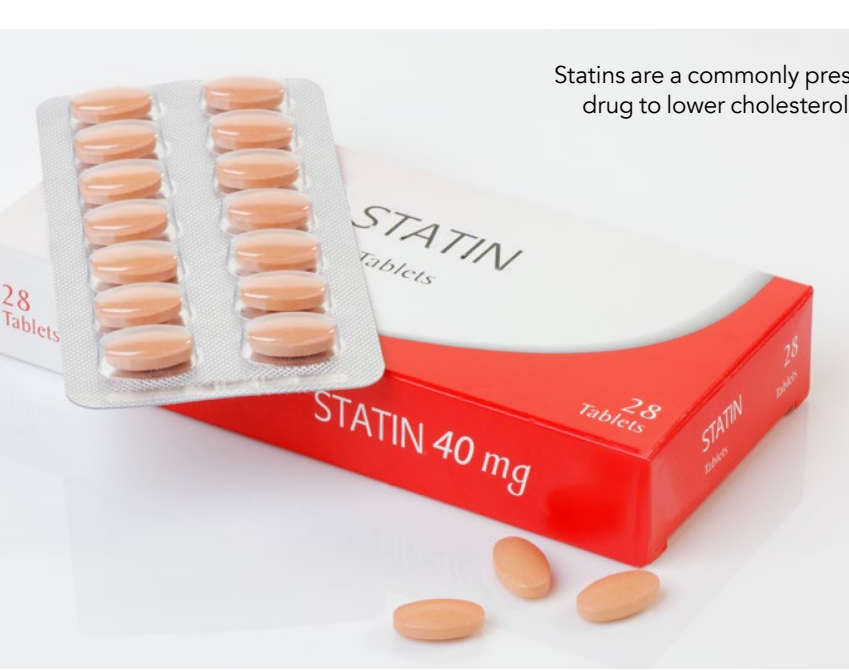

shrouded in mystery. The fact that the biochemistry of HDL metabolism is much more complex than any other lipoprotein doesn't help, but DrVieira answe. "There keen to get to the answer. "There are different types between them, will give us information about the proper function, with regard to cholesterol, of these particles the

tas




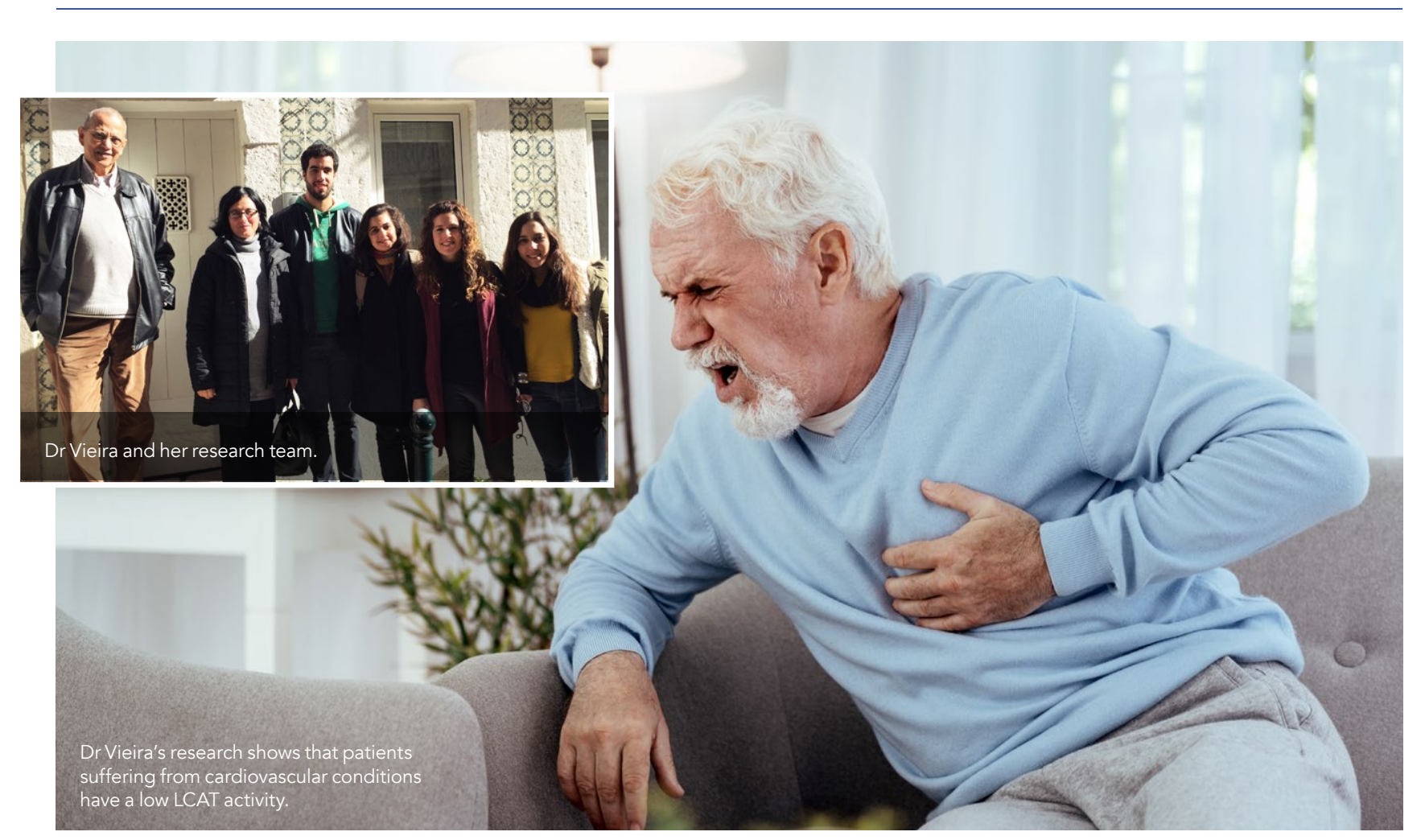

...measuring specific parts of the HDL metabolism - such as LCAT activity - may be more valuable as a diagnostic tool rather than total HDL levels.

\section{In the presence of excessive amounts} of free cholesterol, an enzyme called lecithin-cholesterol transferase (or cholesteryl ester (a more hydrophobic
form of cholesterol). This form is
then safely transported to the liver for elimination.

\section{ATHEROSCLEROSIS}

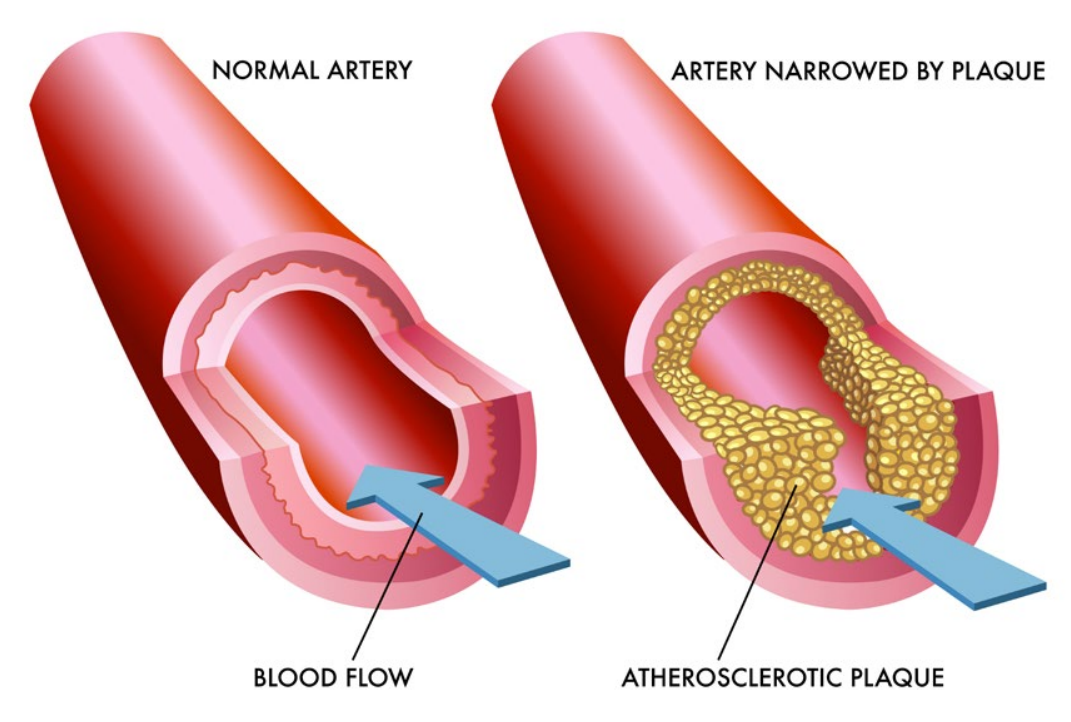

Recently, Dr Vieira showed how this process is affected in patients suffering from cardiovascular conditions due to a low LCAT activity. Comparing a heart attack or ischaemic stroke, the team noticed how the ratio between cholesteryl esters and cholesterol was lower in the patients who had suffere a cardiovascular condition, which could be explained with an inefficien

For the authors, the fact that the activity of LCAT is reduced by about a third this parameter. It seems that measuring specific parts of the HDL metabolism - such as LCAT activity - may be more valuable as a diagnostic tool rather than merely assessing total $\mathrm{HDL}$ levels.

"In this context, the main conclusion of our work is that cardiovascular risk management may be more accurate measurements of HDL metabolism than total HDL and it might also be actually provide information about HDL functionality. Of course, this will have to be confirmed by a prospective cohor
study in the future", concludes Dr Vieira. donors and patients who had suffered suggests a strong predictive value for if risk assessment relies on more precise useful to develop novel biomarkers that

\section{Behind the Research}

\section{Dr Otilia Vieira}

E: otilia.vieira@nms.unl.pt T: +351
human-pathologies-and-infection/

\section{Research Objectives}

Research in Dr Vieira's laboratory aims to better understand the biology, physiology and pathophysiology of

\section{Detail}

CEDOC, NOVA Medical School Faculdade de Ciências Médicas Universidade NOVA Le Lisboa Lortugal

DrVieira completed her PhD in 2000, followed by two post-docs. She performed her 1st post-doc at Grinstein's Lab, Toronto, and the 2nd post-doc at Simons's Lab, MPI-CBG, Dresden. In 2014, she received an iFCT award, at the consolidator level, and moved to CEDOC where she is Principal Investigator.

Funding

PTDC/MED-PAT/29395/2017 financially supported by FCT (Foundation for Science and Technology of the Portuguese Ministry of Science and Higher Education) through national funds and co-funded by FEDER under - NOVAaHealth - UID/M

04462/2013 and the

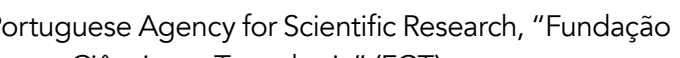
(FCT).

\section{Collaborators}

(Winchil Vaz). - Cardiologists from Hospital Santa Cruz, Carnaxide, Portugal (Manuel S. Almeida, Gustavo Rodrigues, Pedro Araújo-Gonçalves, Jorge Ferreira).

Lipotype GmbH, Tatzberg 47, 01307 Dresden, Germany, Mathias Gerl, Christian Klose, Michal A. Surma and Kai Simons).

ก৩ヘ

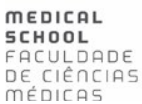

iNOVA4Health

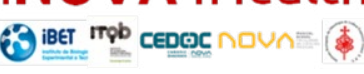

\section{References}

Estronca L, Silva J, Sampaio J, Shevchenko A, Verkade P, Vaz A, Vaz W, Vieira O (2012). Molecular Etiology of Atherogenesis - In Vitro Induction of Lipidosis in Macrophages with a New
LDL Model. PLOS One, 7(4): e34822. https://doi.org/10.3389/ fphys.2018.00654.

Domingues N, Estronca L, Silva J, Encarnação M, Mateus R, Silva D, Santarino I, Saraiva M, Soares M, Pinho e Melo T, lysosome structure and function and induce proinflammatory cytokine production in macrophages. Biochimica et Biophysica Acta 1862, 210-220. https:///doi.org/10.1016/. bbalip.2016.10.009.

Gibson M, Domingues N, Vieira O (2018) Lipid and Non-lipid actors Affecting Macrophage Dysfunction and Inflammation in Atherosclerosis. Front Physiol, 9, 654. https://doi

org/10.3389/fphys.2018.00654.

Gerl M, Vaz W, Domingues N, Klose C, Surma M, Sampaio J, Almeida M, Rodrigues G, Araújo-Gonçalves P, Ferreira J, Borbinha C, Marto J, Vana-Aaplista M, Simons K, Vieira O Esters in the Blood of Cardiovascular Disease Patients. Scientific Reports 8:14764. https://doi.org/10.1038/s41598018-33116-4.

\section{Personal Response}

Do you think this work could lead to a new diagnostic II Yes, we believe that our work will lead to better A more quantitative evaluation of products of exidation of cholesterol and cholesteryl esters in blood may pathogenicity of atheromata in the body. Measurement of LCAT activity in the blood plasma combined with a profiling of $\mathrm{HDL}$ typology may also provide information published so far indicate that more quantitative research along these lines should be encouraged in the near future. 